\title{
Biodiversidad de crustáceos peracáridos en el delta del Río Orinoco, Venezuela
}

\author{
Alberto Martín \& Yusbelly J. Díaz Díaz \\ Universidad Simón Bolívar, Departamento de Estudios Ambientales e Instituto de Tecnología y Ciencias Marinas \\ (INTECMAR). Sartenejas - Baruta. Edo. Miranda. Apdo. Postal 89000. Caracas 1086-A, Venezuela; amartinz@usb. \\ ve; yusdiaz@usb.ve
}

Recibido 08-III-2006. Corregido 26-VI-2006. Aceptado 16-III-2007.

\begin{abstract}
Biodiversity of peracarid crustaceans in the Orinoco River Delta, Venezuela. The composition, abundance, diversity and density of benthonic peracarid crustaceans were studied in the Orinoco river delta, Venezuela. Samples were collected at 18 sites distributed in five areas: one at the high delta, two at the medium sector and two at the low delta. Two field seasons were included: high waters (18-28 November 2002) and low waters (8-19 May 2003). Three samples were dredged at each sampling site, identifying more than 510 specimens belonging to eight families and 11 species: Amphipoda (three families, six species), Isopoda (one family, one species), Tanaidacea (two families, three species), Cumacea (one family) and Mysida (one family, one species). Two species (Discapseudes surinamensis and Halmyrapseudes spaansi) and an unidentified species of Kalliapseudes are new records for Venezuela. Their density and distribution suggest that a salinity gradient influenced longitudinal distribution: greater densities and species richness were in the lower delta, near the mouth of the Mariusa and Winikina rivers. Rev. Biol. Trop. 55 (Suppl. 1): 87-102. Epub 2007 June, 29.
\end{abstract}

Key words: amphipods, isopod, diversity, tanaids, density, Orinoco River.

Las zonas del Golfo de Paria y delta del río Orinoco, constituyen una gran extensión estuarina de gran importancia para la pesca en Venezuela, las cuales recientemente se han convertido en centro del desarrollo de actividades petroleras. Sin embargo, dichas áreas a pesar de poseer un extenso y variado frente costero, han recibido muy poca atención en lo relacionado al estudio sistemático de su biota. Menor aún, ha sido el interés por el estudio de las comunidades bentónicas a pesar de su importancia ecológica, por lo que el conocimiento es escaso, aislado y muy fragmentado, lo que explica la carencia de información consolidada en torno a su distribución, biología y ecología (Drooger y Kaasschieter 1958, Blanco-Belmonte 1989, Díaz 1995, Martín et al. 2002, Bone et al. 2004, Capelo et al. 2004, Pereira et al. 2004).
Igualmente, es de importancia estratégica considerar en el marco referencial, la presencia de varias áreas naturales protegidas, como son los Parques Nacionales Delta del Orinoco o Mariusa, Turuépano y la Reserva de Biosfera Delta del Orinoco, la única de este tipo en el país, y que incluye un área marina, las cuales protegen hábitats de importancia critica para una gran parte de la fauna.

Por otra parte, se ha documentado en la zona de estudio una actividad pesquera intensa sugiriendo de manera potencial, la presencia de comunidades bentónicas utilizadas como recurso alimenticio por la ictiofauna migratoria y residente (Kennish 1986); estando dichas comunidades bentónicas frecuentemente asociadas a fondos blandos fangosos relativamente homogéneos (Capelo et al. 2004). 
En la mayor parte de las publicaciones e informes técnicos consultados se presentan simplemente listas de las especies de organismos, siendo las investigaciones sobre los crustáceos peracáridos (Isopoda, Mysida, Amphipoda, Cumacea y Tanaidacea) muy escasas. Martín et al. (2002), señalan cuatro especies en dos localidades de muestreo: Colomastix bousfieldi, Cerapus thomasi, Apocorophium louisianum y Quadrivisio lutzi. Por otra parte, Capelo et al. (2004), reportan 12 géneros de anfípodos gammarídeos, distribuidos en siete familias, isópodos de siete géneros en seis familias, una especie de cumáceo de la familia Diastylidae, una especie de tanaidáceo (Discapseudes surinamensis) de la familia Parapseudidae y una especie no determinada de la familia Tanaidae.

Cabe destacar la importancia del presente trabajo como contribución al conocimiento de la biodiversidad de crustáceos peracáridos presentes en los sedimentos de la región en el delta del río Orinoco, mediante la evaluación y cuantificación de aspectos como la abundancia, densidad y composición de especies, así como de los posibles patrones espaciales o temporales existentes.

\section{MATERIALES Y MÉTODOS}

Área de estudio. El delta del Orinoco tiene una superficie de unos $40200 \mathrm{~km}^{2}$, de los cuales el propio abanico deltaico ocupa $18810 \mathrm{~km}^{2}$. El delta incluye numerosos caños o brazos que dividen el área en islas. Dado que ocurren fuertes limitaciones en el drenaje de las aguas, existe una aparente similitud de las unidades geomorfológicas que lo conforman. Así se encuentran planicies cenagosas, cubetas o depresiones adosadas a los diques marginales, albardones y complejos de orilla, caños colmatados, marismas e islas de estuarios que bordean los principales ejes de drenajes o caños (Colonnello 2004, Lasso et al. 2004, Méndez 2005 a,b).

El flujo de agua dulce muestra una estacionalidad muy marcada, con una época lluviosa que se extiende desde abril hasta octubre, con el máximo de aguas altas en julio-agosto. La estación seca se extiende desde noviembre a finales de marzo o principios de abril, con el mínimo de aguas bajas entre los meses de febrero a mayo (Lasso et al. 2004). El régimen de marea es semi-diurno y su amplitud varia 1$2 \mathrm{~m}$, haciendo sentir sus efectos hasta unos 200 $\mathrm{km}$ de la costa. Además, las fuertes corrientes de marea permiten que la influencia de las aguas marinas penetre por los caños hasta unos 60-80 km de la costa (Cervigón 1985, Lasso et al. 2004).

Trabajo de campo. Se estudió la macrofauna bentónica, en cinco áreas de muestreo (áreas piloto) (Cuadro 1): una en el alto delta, dos en el sector medio y dos en el bajo delta, las cuales incluyeron, a su vez, 18 estaciones distribuidas de la siguiente forma: alto delta (3 estaciones): Loc-1 (caño Palero), Loc-2 (caño Mariusa-1) y Loc-3 (lagunas inundables); sector medio (7 estaciones): Loc-4 (caño Mariusa-2), Loc-5 (caño Tijitijina o Janakajamana), Loc-6 (caño Jadene o Jajene) y Loc-7 (caño Araguao), Loc-15 (caño Macareo Sur), Loc-16 (caño Macareo Norte) y Loc-17 (caño Guapoa); bajo delta (8 estaciones): Loc-8 (caño Mariusa-3), Loc-9 (caño Caiguara), Loc-10 (laguna/comunidad de Mariusa), Loc-11 (litoral), Loc-12 (isla Tobejuba), Loc-13 (barra Winikina), Loc-14 (Winikina) y Loc-18 (Barra de Mariusita). Se utilizó una draga $\left(0.025 \mathrm{~m}^{2}\right)$, realizándose la toma de tres réplicas para cada estación, durante dos campañas de campo (aguas altas o de lluvia; 18-28 noviembre 2002) y aguas bajas o sequía (08-19 mayo 2003).

Trabajo de laboratorio. El material recolectado fue preservado mediante el uso de formalina neutralizada al $10 \%$ hasta su traslado al laboratorio, para su posterior tamizado mediante tamices de $0.5 \mathrm{~mm}$, previa coloración con rosa de bengala. Luego, con ayuda de pinzas finas y una lupa estereoscópica, se identificaron y cuantificaron todos los crustáceos peracáridos, hasta el menor nivel taxonómico que fuera posible, siguiendo el criterio sistemático del superorden Peracarida reportado por 
CUADRO 1

Coordenadas (UTM) de ubicación de las estaciones de muestreo

TABLE 1

UTM coordinates of the stations sampled

\begin{tabular}{|c|c|c|c|c|}
\hline Área Piloto & Estación & Ubicación & Norte & Este \\
\hline AP1 & 1 & Caño Palero & 978.048 & 623.266 \\
\hline AP1 & 2 & Caño Tortuga & 973.531 & 644.169 \\
\hline AP1 & 3 & Lagunas inundables & 975.763 & 640.561 \\
\hline AP2 & 4 & Caño Mariusa 2 & 1.009 .290 & 675.816 \\
\hline AP2 & 5 & Caño Janakajamana & 1.014 .269 & 697.581 \\
\hline AP2 & 6 & Caño Jadene & 1.007 .545 & 693.708 \\
\hline AP2 & 7 & Caño Araguao & 1.001 .804 & 690.119 \\
\hline AP3 & 8 & Caño Mariusa 3 & 1.042 .500 & 695.001 \\
\hline AP3 & 9 & Caño Caiguara & 1.059 .832 & 697.375 \\
\hline AP3 & 10 & Laguna de Mariusa & 1.065 .479 & 684.988 \\
\hline AP3 & 11 & Litoral de AP3 & 1.065 .529 & 695.117 \\
\hline AP3 & 18 & Barra de Mariusita & 1.061 .469 & 696.326 \\
\hline AP4 & 12 & Isla Tobejuba & 1.027 .907 & 740.638 \\
\hline AP4 & 13 & Barra de Winikina & 1.050 .064 & 724.071 \\
\hline AP4 & 14 & Caño Winikina & 1.043 .350 & 721.138 \\
\hline AP5 & 15 & Caño Macareo Sur & 1.026 .150 & 636.782 \\
\hline AP5 & 16 & Caño Macareo Norte & 1.033 .763 & 640.058 \\
\hline AP5 & 17 & Caño Guapoa & 1.032 .547 & 648.191 \\
\hline
\end{tabular}

Martín y Davis (2001). En el caso del orden Amphipoda se utilizaron las claves taxonómicas especializadas como Barnard y Karaman (1991), Ortiz (1994), Díaz (2001), entre otras. En la identificación del orden Isopoda se utilizó la clave de Kensley y Schotte (1989) y Keable y Bruce (1997); para el orden Tanaidacea se utilizaron las claves de Bacescu y Gûtu (1975), Sieg y Winn (1978) y Gûtu (1996), para el orden Mysida los trabajos de Brattegard (1969, 1970 a,b, 1973, 1974 a,b, 1975) y por último en cuanto al orden Cumacea estos fueron identificados según Kornfield et al. (2001). Las muestras fueron depositadas en la Colección de Crustáceos Peracáridos, Museo de Ciencias Naturales - Universidad Simón Bolívar (USB).

Por otra parte, en cada estación de muestreo, se registraron las condiciones del sedimento (granulometría y materia orgánica total) y la profundidad; así como las características ambientales prevalecientes durante la ejecución del trabajo de campo (Bone et al. 2004), empleando una sonda multiparamétrica (Horiba $\mathrm{U}-10$ ), en donde se evaluaron las siguientes variables: $\mathrm{pH}$, conductividad, oxígeno disuelto, temperatura y salinidad, a los fines de establecer las posibles relaciones con la fauna de peracáridos presente.

Con los datos ambientales y granulométricos obtenidos en cada estación de muestreo durante las dos campañas, se realizaron análisis de componentes principales, utilizando distancia euclidiana simple, previa estandarización de la matriz. Por otra parte, con los datos de abundancia de los crustáceos peracáridos, se realizó una ordenación de las estaciones, utilizando un escalamiento multidimensional no métrico (MDS). Posteriormente se realizaron análisis multivariados de varianzas basados en permutaciones (PERMANOVA) para determinar diferencias significativas $(\mathrm{p}<0.05)$ entre campañas de muestreo y zonas estudiadas. También fueron efectuadas correlaciones entre las variables ambientales y la estructura 
comunitaria. Dichos análisis se realizaron utilizando el paquete estadístico PRIMER.

\section{RESULTADOS}

Se recolectaron e identificaron un total de 515 especimenes pertenecientes a cinco órdenes de crustáceos peracáridos, agrupándose en 8 familias y 11 especies en total, de las cuales dos fueron nuevas citas para el país: Discapseudes surinamensis y Halmyrapseudes spaansi, así como el género Kalliapseudes sp. (Cuadro 2). Al orden Amphipoda correspondieron tres familias y seis especies; en el orden Isopoda se ubicaron una familia y una especie (Natatolana gracilis); Tanaidacea (dos familias, tres especies), Cumacea (una familia, no pudiendo ser identificados a un mayor nivel taxonómico) y Mysida (una familia, una especie).

\section{Listado sistemático de las especies}

Superorden Peracarida Calman, 1904

Orden Amphipoda Latreille, 1816

Suborden Gammaridea Latreille, 1802

Familia Aoridae Walker, 1908

Bemlos kunkelae (Myers, 1977)

Material examinado: estación 13 (1 ९); estación 14 (1 ९). Catálogo: USB (OR-0116, OR-0114).

Distribución y hábitat: Desembocadura del río Surinam, cerca de Paramaribo; Trinidad (Bacescu y Gûtu 1975), Florida (Myers 1977, Camp et al. 1998) y Mar Caribe (Ortíz y Lalana 1998). Marino y estuarino, se encuentra en fondos blandos de tipo fangosos, también presente en algas (Caulerpa sertularioides) en plataformas rocosas litorales, así como en esponjas y en comunidades incrustantes.

Paramicrodeutopus myersi Bynum \& Fox, 1977

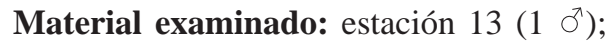
estación 14 (1 †). Catálogo: USB (OR-0117).

Distribución y hábitat: Florida (Myers 1981, Camp et al. 1998) y Mar Caribe (Díaz
2001). Marino, en fondos arenosos hasta $20 \mathrm{~m}$ de profundidad.

Globosolembos smithi (Holmes, 1903)

Material examinado: estación 13 (1 †). Catálogo: se diseccionó por completo al animal y no se catalogó.

Distribución y hábitat: Florida (Thomas 1993, Camp et al. 1998), Golfo de México (Escobar-Briones et al. 2002) y Mar Caribe (Myers 1977, 1981, Atienza 2000, Díaz 2001, Kosoy 2001). Marino, en aguas someras, asociado a fondos con vegetación (praderas de fanerógamas especialmente con algas), esponjas y en la comunidad incrustante de las raíces de mangle.

Familia Ischyroceridae Stebbing, 1899

Cerapus benthophilus Thomas \& Heard, 1979

Material examinado: estación 14 (3 ९). Catálogo: USB (OR-0989).

Distribución y hábitat: Golfo de México (Thomas y Heard 1979, Ledoyer 1986, Winfield y Ortíz 1996, Winfield et al. 1997) y Florida (Thomas y Heard 1979, Camp et al. 1998). Marino, en sistemas lagunar-estuarinos, construye tubos en fondos limosos - fangosos y asociados a fanerógamas marinas.

Familia Melitidae Bousfield, 1973

Eriopisa incisa McKinney, Kalke \& Holland, 1978

Material examinado: estación 10 (1 †). Catálogo: USB (OR-0115).

Distribución y hábitat: Golfo de México (Escobar-Briones et al. 2002) y Mar Caribe (McKinney et al. 1978, Ortíz y Lalana 1993, Díaz 2001, Díaz y Martín 2001). Marino, en fondos arenosos.

Anamaera hixoni Thomas \& Barnard, 1985

Material examinado: estación 5 (1 ९). Catálogo: USB (OR-0987).

Distribución y hábitat: Florida (Thomas y Barnard 1985, Thomas 1993, Camp et al. 1998). Marino, en zonas rocosas adheridos a algas, entre 1 y $2 \mathrm{~m}$ de profundidad. 


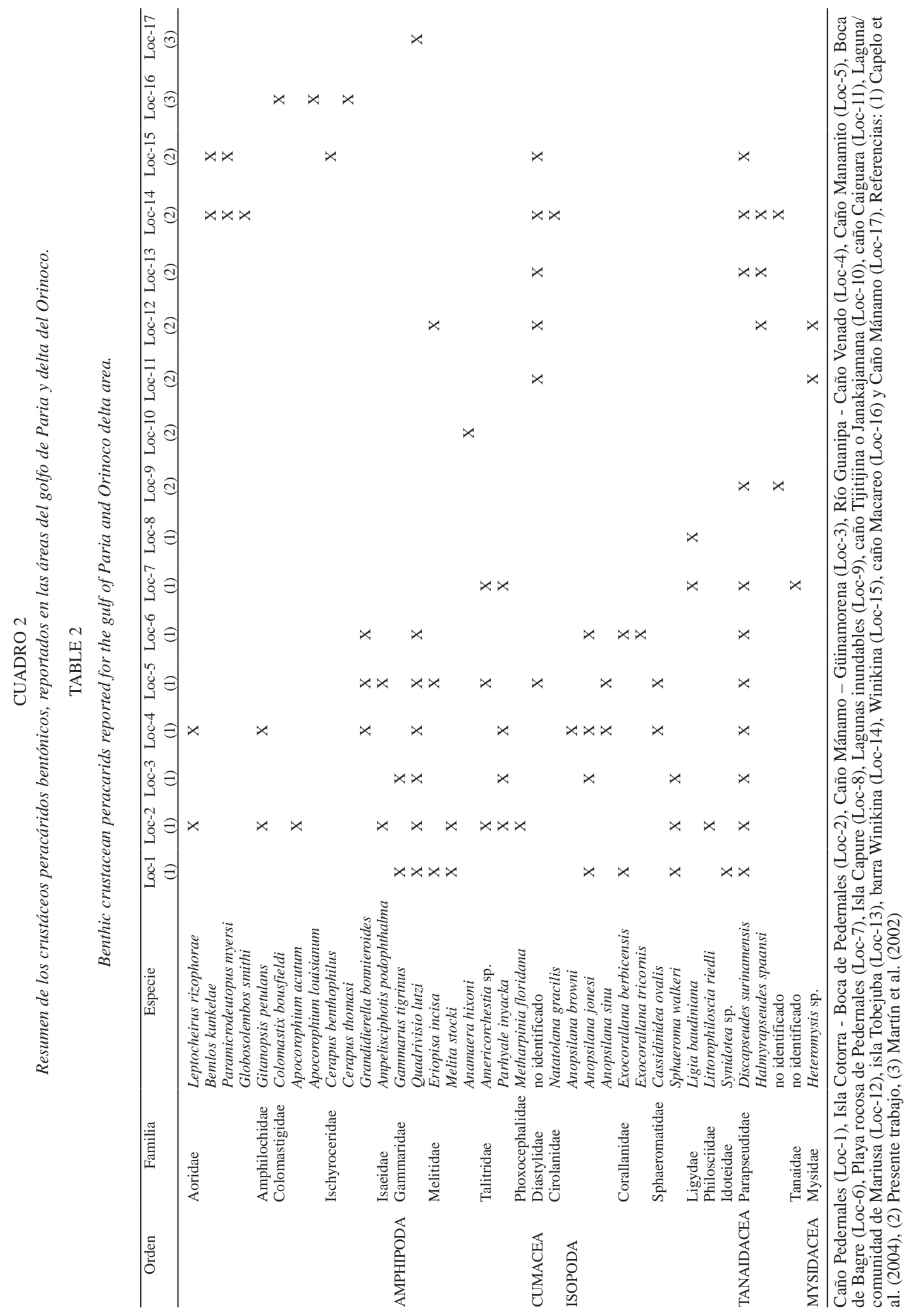


Orden Isopoda Latreille, 1817

Suborden Flabellifera Sars, 1882

Familia Cirolanidae Dana, 1852

Natatolana gracilis (Hansen, 1890)

Material examinado: un ejemplar sin sexar: estación 13. Catálogo: USB (ISO-0777).

Distribución y hábitat: San Thomas (Islas Vírgenes), costas de Florida, norte de Brasil. Se ha reportado hasta $120 \mathrm{~m}$ de profundidad (Kensley y Schotte 1989, Keable y Bruce 1997).

Orden Tanaidacea Dana, 1849

Material examinado: estación 3 (4 sin sexar); estación 13 (1 sin sexar). No pudieron ser identificados, dado su mal estado y pequeño tamaño. Catálogo: USB (TA-0641, TA-0654, TA-0664).

Suborden Apseudomorpha Sieg, 1980

Familia Parapseudidae Gûtu, 1981

Discapseudes surinamensis Bacescu \& Gûtu, 1975

Material examinado: Se identificaron 389 ejemplares: estación 3 (4 sin sexar); estación 12 (6 $\uparrow, 2$ o 1 juvenil); estación 13 (193, 32 ovadas; 30 ○, 44 juveniles, 4 sin sexar); estación 14 (61 9,3 ovadas; $21{ }^{\star}$, 22 juveniles, 1 sin sexar). Catálogo: USB (TA-0571, TA-0645, TA-0646, TA-0653, TA-0656, TA-0657, TA-0658, TA0659, TA-0660, TA-0661, TA-0665, TA-0666, TA-0667, TA-0668, TA-0669, TA-0670, TA0671, TA-0672, TA-0673, TA-0674).

Distribución: Desembocadura del río Surinam, cerca de Paramaribo; Trinidad (Bacescu y Gûtu 1975). Marino y estuarino, se encuentra en fondos blandos de tipo fangosos.

Halmyrapseudes spaansi Bacescu \& Gûtu, 1975

Material examinado: Se identificaron 29 ejemplares: estación 10 (14 9,5 ơ, 2 juveniles); estación 12 (1 $\sigma^{\top}, 1$ sin sexar); estación 13 (2 \&, 3 o', 1 sin sexar). Catálogo: USB (TA-0572, TA-0644, TA-0647, TA-0648, TA-0649, TA0650, TA-0651, TA-0652, TA-0655, TA0662).
Distribución y hábitat: Desembocadura del río Surinam, cerca de Paramaribo; Trinidad (Bacescu y Gûtu 1975). Marino y estuarino, se encuentra en fondos blandos de tipo fangosos.

Orden Cumacea Kröyer, 1846

Familia Diastylidae Bate, 1856

Material examinado: Se identificaron 63 ejemplares: estación 9 (1 sin sexar); estación 10 (2 ơ); estación 12 (6 sin sexar); estación 13 (46 sin sexar); estación 14 (1 \&, ovada; 7 sin sexar). Catálogo: USB (CU-0332, CU-0333, CU-0334, CU-0335, CU-0336, CU-0337, CU0338, CU-0339, CU-0340, CU-0342, CU-0343, CU-0344, CU-0345, CU-0346, CU-0347, CU0348, CU-0349, CU-0350, CU-0351, CU-0352, CU-0353, CU-0354, CU-0355). No pudieron ser identificados, dado su pequeño tamaño.

Orden Mysida Haworth, 1825

Familia Mysidae Haworth, 1825

Heteromysis sp. Smith, 1873

Material examinado: Se identificaron 20 ejemplares: estación 9 (4 sin sexar); estación 10 (16 sin sexar). Catálogo: USB (MYS0327, MYS-0328, MYS-0329, MYS-0330). No pudieron ser identificados, dado su pequeño tamaño.

Variación temporal. Se recolectaron 425 ejemplares provenientes del muestreo de aguas altas (82.5 \%) y 90 en aguas bajas (17.5\%), en donde los tanaidáceos presentaron el mayor valor (81.8\%), seguidos por los cumáceos (12.2\%), misidáceos (3.9\%), anfípodos $0.2 \%$ e isópodos (0.002 \%).

En general, los peracáridos fueron más abundantes en la campaña de aguas altas (64.69 ind $\mathrm{m}^{-2}$ ), en comparación con la campaña de aguas bajas (38.46 ind $\mathrm{m}^{-2}$ ) (Cuadros 3 y 4). Por otra parte, los isópodos y misidáceos sólo se encontraron en la época de aguas altas. En el caso de los anfípodos, la familia Ischyroceridae (46.3\%) fue la más abundante debido a la aparición en grandes densidades de la especie Cerapus benthophilus durante la época de aguas bajas, seguida por la familia Aoridae (25.7 \%) debido a la gran abundancia de la 
CUADRO 3

Densidad promedio (ind $\mathrm{m}^{-2}$ ) de crustáceos peracáridos en la campaña de aguas altas

TABLE 3

Average density (ind $\mathrm{m}^{-2}$ ) of peracarid crustaceans for high waters period

$\begin{array}{ccccccc}\text { Orden } & \text { AP-1 } & \text { AP-2 } & \text { AP-3 } & \text { AP-4 } & \text { AP-5 } & \text { Promedio } \\ \text { Amphipoda } & 0.00 & 0.00 & 0.89 & 3.70 & 0.00 & 0.92 \\ \text { Tanaidacea } & 6.67 & 2.22 & 0.89 & 256.30 & 0.00 & 53.21 \\ \text { Cumacea } & 0.00 & 0.00 & 1.33 & 37.78 & 0.00 & 7.82 \\ \text { Mysidacea } & 0.00 & 0.00 & 12.44 & 0.00 & 0.00 & 2.49 \\ \text { Isopoda } & 0.00 & 0.00 & 0.00 & 1.23 & 0.00 & 0.25 \\ \text { Total } & 6.67 & 2.22 & 15.55 & 299.01 & 0.00 & 64.69\end{array}$

CUADRO 4

Densidad promedio (ind $\mathrm{m}^{-2}$ ) de crustáceos peracáridos en la campaña de aguas bajas

TABLE 4

Average density (ind $\mathrm{m}^{-2}$ ) of peracarid crustaceans for low waters period

$\begin{array}{ccccccc}\text { Orden } & \text { AP-1 } & \text { AP-2 } & \text { AP-3 } & \text { AP-4 } & \text { AP-5 } & \text { Promedio } \\ \text { Amphipoda } & 0.00 & 1.67 & 0.00 & 6.67 & 1.48 & 1.96 \\ \text { Tanaidacea } & 4.44 & 0.00 & 19.56 & 92.59 & 2.22 & 23.76 \\ \text { Cumacea } & 0.00 & 0.00 & 0.00 & 63.70 & 0.00 & 12.74 \\ \text { Mysidacea } & 0.00 & 0.00 & 0.00 & 0.00 & 0.00 & 0.00 \\ \text { Isopoda } & 0.00 & 0.00 & 0.00 & 0.00 & 0.00 & 0.00 \\ \text { Total } & 4.44 & 1.67 & 19.56 & 162.96 & 3.70 & 38.46\end{array}$

especie Bemlos kunkelae, durante la época de aguas altas. La familia Parapseudidae representó el $97.5 \%$ de los tanaidáceos encontrados (Discapseudes surinamensis).

En cuanto a la densidad promedio, los tanaidáceos representaron el grupo con los mayores valores (38.49 ind $\mathrm{m}^{-2}$ ), seguidos por los cumáceos (10.28 ind $\left.\mathrm{m}^{-2}\right)$, los anfípodos (1.44 ind $\left.\mathrm{m}^{-2}\right)$, los misidáceos $\left(1.25\right.$ ind $\left.\mathrm{m}^{-2}\right) \mathrm{y}$ los isópodos (0.12 ind $\mathrm{m}^{-2}$ ) (Cuadro 5). En la época de aguas altas, los tanaidáceos registraron 53.21 ind $\mathrm{m}^{-2}$, mientras que en la época de

\section{CUADRO 5}

Densidad promedio (ind $\mathrm{m}^{-2}$ ) de crustáceos peracáridos en ambas campañas de muestreo

TABLE 5

Average density (ind $\mathrm{m}^{-2}$ ) of peracarid crustaceans for both periods

$\begin{array}{ccccccc}\text { Orden } & \text { AP-1 } & \text { AP-2 } & \text { AP-3 } & \text { AP-4 } & \text { AP-5 } & \text { Promedio } \\ \text { Amphipoda } & 0.00 & 0.84 & 0.45 & 5.19 & 0.74 & 1.44 \\ \text { Tanaidacea } & 5.56 & 1.11 & 10.23 & 174.45 & 1.11 & 38.49 \\ \text { Cumacea } & 0.00 & 0.00 & 0.67 & 50.74 & 0.00 & 10.28 \\ \text { Mysidacea } & 0.00 & 0.00 & 6.22 & 0.00 & 0.00 & 1.25 \\ \text { Isopoda } & 0.00 & 0.00 & 0.00 & 0.62 & 0.00 & 0.12 \\ \text { Total } & 5.56 & 1.95 & 17.57 & 231.00 & 1.85 & 51.58\end{array}$


aguas bajas, se encontró un valor mucho menor (23.76 ind $\mathrm{m}^{-2}$ ).

Al comparar la estructura comunitaria de los peracáridos, en las dos campañas de muestreo realizadas, mediante una ordenación MDS y un análisis de similaridad (ANOSIM), no se encontró ninguna separación significativa entre ambas campaña de muestreo $(\mathrm{p}=0.635)$ (Fig. 1).

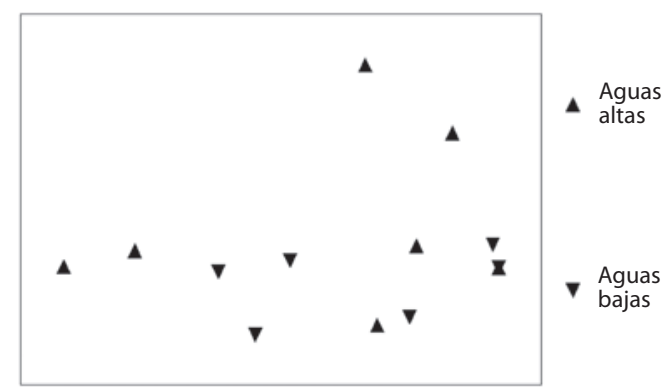

Fig. 1. Ordenación MDS de los crustáceos peracáridos presentes en ambas épocas de muestreo.

Fig. 1. MDS plot of peracarid crustaceans for both sampling periods.

Parámetros ambientales. En general, los resultados obtenidos indican valores de salinidad y conductividad muy bajos en todas las estaciones muestreadas, presentando una clara dominancia del régimen fluvial del río Orinoco en aguas altas. En todas las estaciones se presentaron aguas ligeramente ácidas, con un valor de $\mathrm{pH}$ promedio de 5.72, variando entre un máximo de 6.65 (litoral de bajo delta) y un mínimo de 4.91 (Caño Guapoa, sector medio) (Cuadro 6).

La concentración de oxígeno disuelto y la temperatura del agua, fueron variables, pasando por aguas casi anóxicas en las estaciones 4 y 17 (sector medio). Por otra parte, los mayores valores se observan en las estaciones 12 (bajo delta), 15 y 7 (sector medio), promediando en dichas estaciones $6.60 \mathrm{mg} \mathrm{l}^{-1}$. En cuanto a la temperatura del agua, varió aproximadamente unos $5{ }^{\circ} \mathrm{C}$ entre máximas y mínimas entre todas las 18 estaciones evaluadas (Cuadro 6).

Los sedimentos de las estaciones estudiadas mantuvieron una granulometría dominada por las fracciones finas, con clara dominancia de los fangos, con un promedio de $59.8 \%$ sobre el total del sedimento. El contenido de materia orgánica en las muestras vario ampliamente, desde un máximo de $26.5 \%$ en la estación Lagunas inundables (alto delta), hasta un mínimo de $2.6 \%$, en Caño Palero (alto delta) (Cuadro 6).

En aguas bajas, los resultados obtenidos indican valores de salinidad y conductividad con una mayor influencia salina en varias estaciones, particularmente en las estaciones 11 (litoral de bajo delta), con $44.52 \mathrm{mS} \mathrm{cm}^{-1}$ para conductividad y $28.8 \%$ de salinidad, respectivamente, así como en las estaciones 3 (Lagunas inundables, alto delta), 8 (Caño Mariusa 3, bajo delta) y 9 (Caño Caiguara, bajo delta), evidenciando el menor nivel de las aguas dulces del río Orinoco. En el resto de las estaciones se mantuvo la clara influencia del río y su sistema de drenaje. En general, todas las estaciones presentaron aguas ligeramente ácidas, con un valor de $\mathrm{pH}$ promedio de 6.80; variando entre un máximo de 7.97 en la estación 11 (litoral de bajo delta) y mínimos de 5.81 Caño Janakajamana (sector medio) y de 5.97 en la estación de Caño Guapoa (sector medio) (Cuadro 7).

El oxígeno disuelto mostró en general valores superiores al muestreo efectuado en aguas altas, con un valor promedio de 6.79 $\mathrm{mg} \mathrm{l}^{-1}$, variando entre un máximo de 7.82 $\mathrm{mg} \mathrm{l}^{-1}$ en la estación 3 Lagunas inundables, alto delta) y un mínimo de $3.95 \mathrm{mg} \mathrm{l}^{-1}$ en la estación 5 de Caño Janakajamana (sector medio). Por otra parte, la temperatura mostró una variación de apenas $2{ }^{\circ} \mathrm{C}$ entre valores máximos y mínimos, con un promedio de $28.16{ }^{\circ} \mathrm{C}$ (Cuadro 7).

Los fangos nuevamente representaron la fracción del sedimento con mayor dominancia en el área del estudio, con un promedio de $76.1 \%$, lo que muestra un importante incremento en relación con la campaña precedente (59.8\%). Por su parte, la variación en el contenido de materia orgánica en las muestras fue mucho menor, manteniéndose un valor promedio similar entre $7.3 \%$ y $7.8 \%$ en ambas campañas (Cuadros 6 y 7 ). 

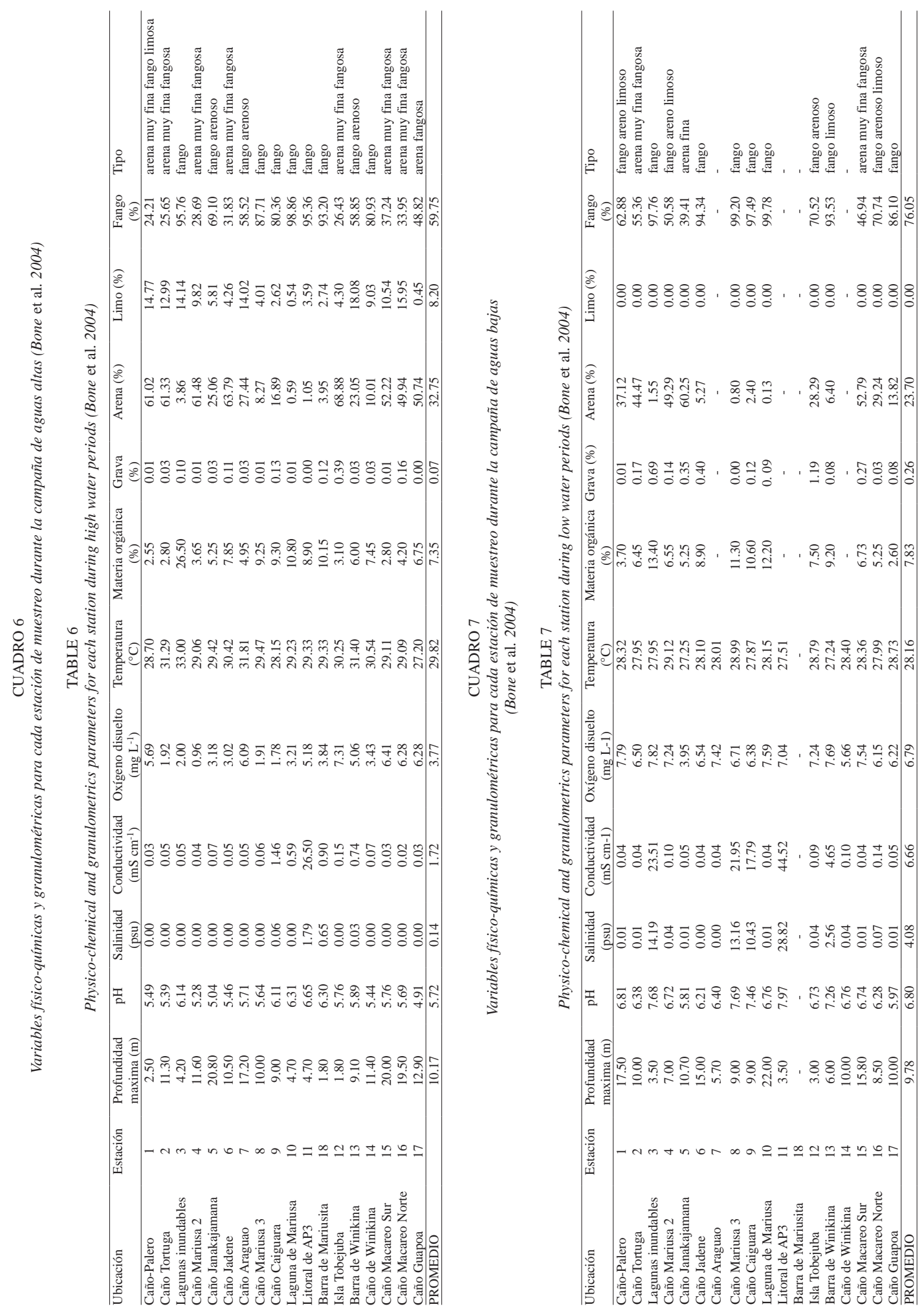
Las variaciones antes señaladas pueden evidenciarse en el análisis de componentes principales (PCA) realizado, obteniéndose en los dos primeros ejes de ordenación el $60 \%$ de la variación, donde el componente 1, explica el $41 \%$, debido principalmente a parámetros fisicoquímicos como el $\mathrm{pH}$ (r Spearman=0.434) y la salinidad (r Spearman $=0.388$ ), y a variables granulométricas como el porcentaje de fango (r Spearman= 0.407). En el componente 2 se explica el $19 \%$ de la variación, donde las variables más importantes fueron el oxígeno disuelto (r Spearman $=-0.508$ ), la temperatura (r Spearman= 0.444) y el porcentaje de materia orgánica (r Spearman= 0.427) $($ Fig. 2).

Variación espacial. En cuanto a la distribución espacial de los peracáridos dentro de las áreas piloto muestreadas, se observaron diferencias en la composición y densidad de las especies. Área piloto 1: únicamente en la estación 3 (Lagunas inundables) se encontraron tanaidáceos del género Kalliapseudes sp., correspondiendo a su mayor densidad (16.67 ind $\mathrm{m}^{-2}$ ). Área piloto 2: solo en esta área piloto se encontraron anfípodos de la especie Anamaera hixoni. Asimismo, se encontraron anfípodos (3.33 ind $\mathrm{m}^{-2}$ ) de la familia Melitidae (solo en la estación 5-Janakajamana) y tanaidáceos de la familia Parapseudidae (Discapseudes surinamensis), únicamente en la estación 4 (Caño Mariusa), en densidades de 4.44 ind $\mathrm{m}^{-2}$. Área piloto 3: se encontraron anfípodos (2.22 ind $\mathrm{m}^{-2}$ ) de la familia

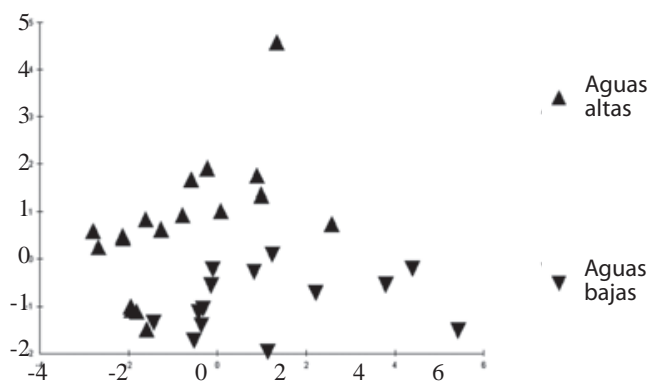

Fig. 2. Análisis de componentes principales (ACP) de los parámetros ambientales para ambas épocas de muestreo.

Fig. 2. Principle Component Analysis (PCA) plot of environmental parameters for both sampling periods.
Melitidae en la estación 10 (Laguna/comunidad de Mariusa), misidáceos (Mysidae) en las estaciones 9 (Caiguara) y 10 (17.78 ind $\mathrm{m}^{-2}$ ), tanaidáceos (Parapseudidae) (51.11 ind $\mathrm{m}^{-2}$ ) nuevamente en la estación 10 y finalmente los cumáceos (estación 9), en densidades de 3.33 ind $\mathrm{m}^{-2}$. Área piloto 4: los tanaidáceos (Parapseudidae) fueron los peracáridos más abundantes en promedio para las estaciones ubicadas en dicha área (174.44 ind $\mathrm{m}^{-2}$ ), representando el $86.4 \%$ de todos los peracáridos colectados en la estación 12 (isla Tobejuba), seguidos por los cumáceos (11.0\%). En la estación 13 (Barra de Winikina) los tanaidáceos representaron el 91.7 \% y en la estación 14 (Winikina) el 68.9 \%. También se encontraron anfípodos de las familias Aoridae e Ischyroceridae, solamente en las estaciones 13 y 14 , con densidades que oscilaron entre 2.22 ind $\mathrm{m}^{-2}$ y 13.33 ind $\mathrm{m}^{-2}$ respectivamente. Área piloto 5: sólo se encontraron anfípodos en la estación 15 (Caño Macareo Sur) con densidades de 2.22 ind $\mathrm{m}^{-2} \mathrm{y}$ tanaidáceos (3.33 ind $\mathrm{m}^{-}$ ${ }^{2}$ ) en la estación 16 (Caño Macareo Norte).

Sin embargo, cuando se realizó la ordenación multidimensional no métrica (MDS) de la comunidad de peracáridos por campaña de muestreo y áreas piloto, dichas diferencias de composición de especies no fueron significativas. El análisis de similaridad (ANOSIM) entre las áreas piloto para cada campaña de muestreo, demostró que no existen diferencias significativas entre las áreas piloto 1,2 y 5 , ni entre las áreas piloto 3 y $4(\mathrm{p}=0.05)$, conformándose entonces sólo dos grupos de estaciones: un grupo que refleja una zona dominada por la influencia fluvial (áreas piloto 1,2 y 5) y otro grupo que indica una zona de influencia oceánica (áreas piloto 3 y 4) $(\mathrm{p}=0.002)$. De esta manera, al realizar la ordenación de la comunidad (MDS) con estas nuevas zonas para cada campaña de muestreo, se observa una diferencia tanto en aguas altas $(\mathrm{p}=0.013)$, como en aguas bajas $(\mathrm{p}=0.029)$ de la estructura comunitaria de los peracáridos, en las estaciones de la zona de influencia fluvial con respecto a las estaciones de influencia oceánica (Fig. 3 A, B). 

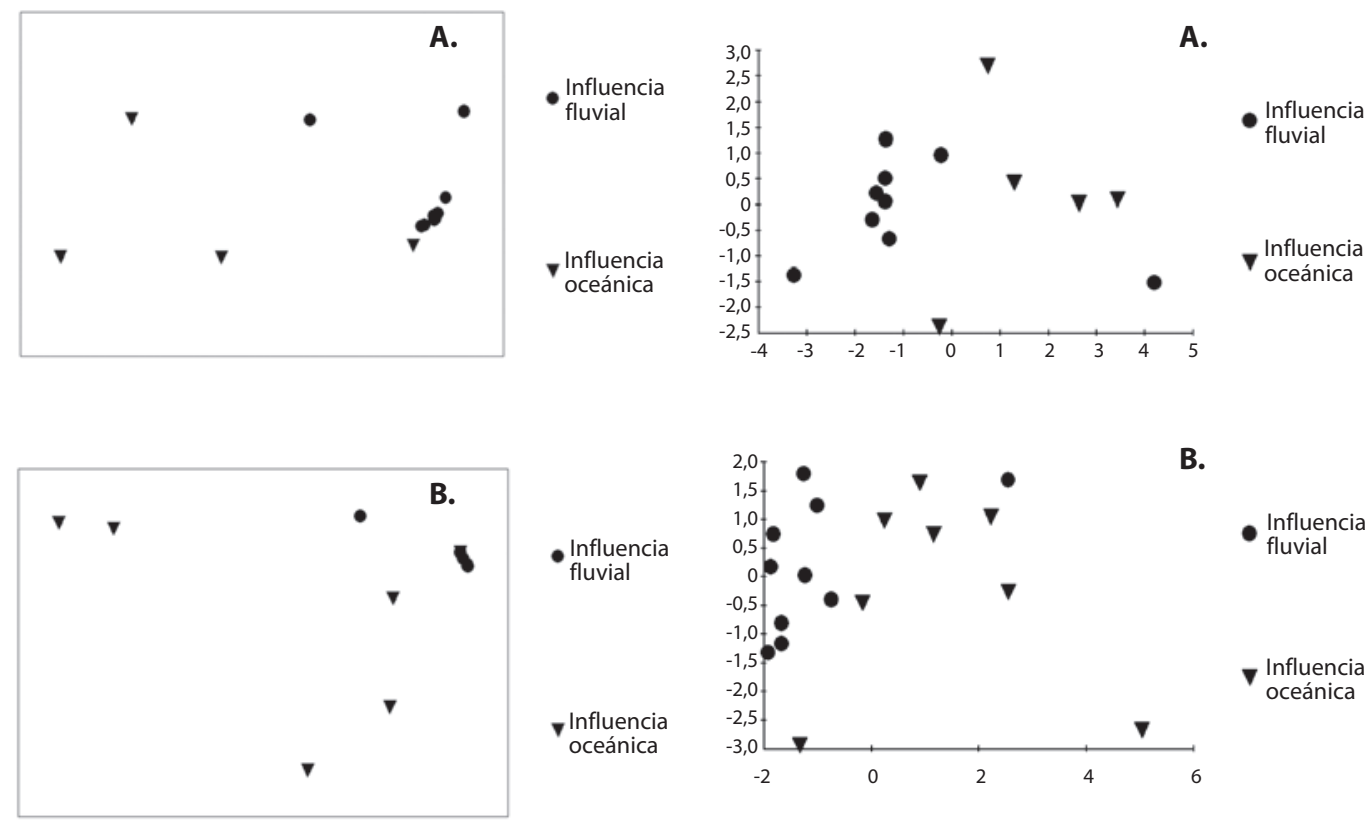

Fig. 3. Ordenación MDS de los peracáridos presentes en cada época de muestreo. A. aguas bajas, B. aguas altas. Fig. 3. MDS plot of peracarids for each sampling period. A. low waters, B. high waters.

Esta ordenación de las zonas es debida fundamentalmente a las mayores abundancias de tanaidáceos y cumáceos en la zona de influencia litoral, para el muestreo en aguas bajas; mientras que en aguas altas, la ordenación está definida por las mayores abundancias de tanaidáceos, cumáceos y misidáceos, en dicha zona, principalmente en las estaciones 13 y 14 .

Parámetros ambientales. En el caso de las variables ambientales, también se agruparon las áreas piloto en dos grandes grupos debido a las similaridades entre las mismas, obteniéndose una separación entre las estaciones de la zona de influencia fluvial, de las estaciones de influencia oceánica para cada campaña de muestreo (Fig. 4 A, B).

En aguas bajas, las estaciones con influencia oceánica, están relacionadas con el componente 1 (salinidad, $\mathrm{pH}$ y conductividad), mientras que las estaciones bajo influencia

Fig. 4. Análisis de components principales (ACP) de los parámetros ambientales para cada época de muestreo. A. aguas bajas, B. aguas altas.

Fig. 4. Principle Component Analysis (PCA) plot of environmental parameters for each sampling period. A. low waters, B. high waters.

fluvial están más relacionadas con el componente 2 (profundidad máxima y negativamente con el porcentaje de grava). En aguas altas, por su parte, las estaciones de mayor influencia oceánica, están relacionadas con el porcentaje de fango, mientras que las estaciones bajo influencia fluvial, están relacionadas negativamente con la concentración de oxígeno disuelto, la conductividad y la salinidad.

\section{DISCUSIÓN}

Ampliaciones de ámbito. En el caso de los anfípodos, las familias Aoridae, Ischyroceridae y Melitidae ya habían sido reportadas anteriormente en la zona de Pedernales, pero no se habían hecho la identificación hasta el nivel de especie, siendo las seis especies encontradas en esta investigación (Bemlos kunkelae, Paramicrodeutopus myersi, Globosolembos smithi, Cerapus benthophilus, Eriopisa incisa 
y Anamaera hixoni), nuevos registros para la zona ampliándose los rangos de distribución, particularmente en el área de golfo de Paria y delta del Orinoco.

Entre los peracáridos identificados, el grupo de los tanaidáceos fue reportado anteriormente en el delta, para el caño Mariusa y Punta Pescador, pero las especies Discapseudes surinamensis, Halmyrapseudes spaansi y el género Kalliapseudes sp. no habían sido señaladas anteriormente para Venezuela. Los cumáceos también habían sido reportados como grupo en la zona de Pedernales.

Variación temporal. Aún cuando la estructura comunitaria de los peracáridos no supone una variación temporal significativa, es posible observar mayores densidades de tanaidáceos y misidáceos en aguas altas, tal vez por ser estos organismos más eurihalinos, como lo señala Castello (1985).

Aunque los anfípodos no fueron un grupo determinante en la disimilaridad entre campañas de muestreo (aguas altas - aguas bajas), su composición varió marcadamente. En la época de aguas bajas, la especie Cerapus benthophilus fue la más abundante, mientras que en la época de aguas altas fue Bemlos kunkelae, probablemente por sus diferentes formas de vida. C. benthophilus es una especie que construye tubos en fondos limosos-fangosos en sistemas lagunar-estuarinos, como son predominantemente las estaciones con influencia fluvial en aguas bajas; mientras que $B$. kunkelae es una especie principalmente marina (Myers 1977, Thomas y Heard 1979), por lo cual no es extraño encontrarla en las estaciones con influencia oceánica cercanas a la línea de costa del delta.

Parámetros ambientales. En líneas generales, las estaciones estudiadas mantuvieron una composición textural dominada por las fracciones finas, con clara dominancia de los fangos. Los valores de salinidad y conductividad presentaron una mayor influencia salina en varias estaciones, particularmente durante la campaña de aguas bajas, evidenciando el menor nivel y velocidad de las aguas dulces del río Orinoco y su efecto deposicional (Méndez 2005a), particularmente en los tramos finales de su recorrido.

Variación espacial. De acuerdo con los resultados de la densidad de peracáridos, el área piloto 4 resultó ser la más importante en términos de densidad. La densidad y distribución de estos organismos en la región del delta del Orinoco muestreada, parece indicar la existencia de gradientes de salinidad, la presencia de una mayor densidad y riqueza de especies en las áreas piloto 3 y 4, que posteriormente se agruparon en la zona de influencia oceánica, puede estar relacionada al mayor efecto marino a la que se encuentran sometidas estas áreas, restringiendo la distribución de los organismos en algunos casos, dado que varios grupos de peracáridos solo se encontraron en dicha zona.

La macrofauna encontrada en el delta del Orinoco, fue bastante menos diversa en términos de riqueza de especies con respecto a lo reportado para las aguas atlánticas de Venezuela, en donde se identificaron ocho especies de anfípodos, cuatro cumáceos, ocho isópodos y cinco tanaidáceos (Martín y Díaz 2005); aunque cabe destacar que dicha macrofauna atlántica se corresponde con ambientes netamente marinos en un amplio gradiente de profundidad, no encontrándose ninguna especie en común, en dichas áreas.

Capelo et al. (2004), señalan que en cuanto al número de especies, los crustáceos peracáridos representaron el $42 \%$ de la biota bentónica registrada, en donde la especie Discapseudes surinamensis fue la mayormente distribuida (al igual que en el presente estudio), encontrándose en siete de las ocho localidades muestreadas. En orden de importancia le siguieron: Quadrivisio lutzi en seis localidades y Parhyale inyacka y Anopsilana jonesi en cuatro localidades. En general, se encontró una alta riqueza y diversidad de especies bentónicas, a pesar de ser esta una región estuarina. Esto se debe principalmente a que confluyen especies típicamente marinas como: Gitanopsis petulans, Ampelisciphotis podophthalma, Eriopisa incisa, Exocorallana tricornis, Ligia baudiniana, 
Metharpinia floridana y Synidotea sp.; especies estuarinas como Grandidierella bonnieroides, Gammarus tigrinus, Anopsilana jonesi y A. sinu y especies típicas de agua dulce como Anopsilana browni y Exocorallana berbicensis (Capelo et al. 2004).

Las comunidades bentónicas de la zona del golfo de Paria y delta del Orinoco se presentan asociadas a ambientes muy homogéneos y con una alta repetitividad, lo que permite una gran oportunidad para la conservación del área; ya que las zonas no perturbadas podrían servir como reservorio o refugio para otras regiones con impacto ambiental (Capelo et al. 2004).

Esta investigación, viene a complementar la escasa literatura sobre crustáceos peracáridos en la zona, por lo que el presente aporte sistemático constituye un fortalecimiento hacia el conocimiento del grupo en esta región del delta del río Orinoco, adicionalmente a los ya realizados por Martín et al. (2002) y Capelo et al. (2004).

Parámetros ambientales. En general los resultados obtenidos indican valores de salinidad y conductividad bajos en la mayoría de las estaciones muestreadas (dominancia del régimen fluvial), sin embargo se pudo apreciar unas claras influencias salinas en las áreas piloto 3 y 4 (zona de influencia oceánica), ubicadas en áreas del delta inferior, de mayor influencia intermareal. Méndez (2005a), señala que las facies del delta inferior, básicamente en una franja relativamente angosta de aproximadamente $10 \mathrm{~km}$ de ancho, están relacionadas principalmente con el oleaje, corrientes marinas y el nivel de las mareas, en donde los procesos sedimentarios más importantes están formando facies de lomas de playa, facies de acreción de bancos de sedimentos finos, así como ambientes de marismas.

Desde la boca de Mariusita hasta Punta Pescadores, en la desembocadura del Macareo, la progradación, moldeada por el sistema de corrientes y el oleaje, está desarrollando acumulaciones importantes de arena fina, muy fina y material limo-arcilloso Méndez (2005a), las cuales resultan en hábitats potenciales para el desarrollo de una muy diversa fauna bentónica, capaz de soportar los drásticos cambios en la salinidad.

\section{AGRADECIMIENTOS}

Este trabajo forma parte integral del proyecto VEN/99/G31 “Conservación y uso sustentable de la diversidad biológica en la reserva de biosfera y los humedales del Delta del Orinoco, evaluación ecológica rápida de la fauna acuática”, financiado por el PNUDMARN (Oficina Nacional de Diversidad Biológica). Nuestro agradecimiento a Juan José Cruz y Eduardo Klein por su ayuda en los análisis de los datos.

\section{RESUMEN}

Para evaluar la composición, abundancia, diversidad y densidad de crustáceos peracáridos en los sedimentos de la región del delta del río Orinoco (Venezuela), establecimos cinco áreas de muestreo: una en el alto delta, dos en el sector medio y dos en el bajo delta (18 estaciones). Hicimos dos muestreos de campo: época de "aguas altas" o lluvia (18-28 noviembre/2002) y “aguas bajas” o sequía (8-19 de mayo, 2003). Tomamos tres muestras de bentos mediante dragas, identificando un total de 515 individuos, pertenecientes a 8 familias y 11 especies en total: Amphipoda (tres familias, seis especies), Isopoda (una familia, una especie), Tanaidacea (dos familias, tres especies), Cumacea (una familia) y Mysida (una familia, una especie), de las cuales dos fueron nuevas citas para el país: Discapseudes surinamensis y Halmyrapseudes spaansi, así como una especie no identificada de Kalliapseudes. La densidad y distribución de estos organismos sugiere la existencia de gradientes de influencia marina, con las mayores densidades y riqueza de especies en áreas cercanas a las desembocaduras de los caños Mariusa y Winikina.

Palabras clave: anfípodos, isópodos, diversidad, tanaidáceos, densidad, río Orinoco.

\section{REFERENCIAS}

Atienza, D. 2000. Taxonomía y ecología de los anfípodos asociados a praderas de Thalassia testudinum en el Parque Nacional Morrocoy. Tesis de Licenciatura, Universidad Simón Bolívar, Caracas, Venezuela. 129 p. 
Bacescu, M. \& M. Gûtu. 1975. A new genus (Discapseudes n.g.) and three new species of Apseudidae (Crustacea, Tanaidacea) from the northeastern coast of South America. Zool. Meded. 49: 95-113.

Barnard, J.L. \& G.S. Karaman. 1991. The families and genera of marine Gammaridean Amphipoda (Except marine Gammaroids). Record Aust. Mus. 13: 1- 866.

Blanco-Belmonte, L. 1989. Estudio de las comunidades de invertebrados asociados a las macrófitas acuáticas de tres lagunas de inundación de la sección baja del río Orinoco, Venezuela. Mem. Soc. Cienc. Nat. La Salle. 49: 71-107.

Bone, D., A. Machado, P. Spiniello, M. Ortaz, J. Posada, R. Molinet, E. Yerena, C. Rodríguez, E. Klein \& A. Martín (eds.). 2004. Conservación y uso sustentable de la diversidad biológica en la reserva de biosfera y los humedales del delta del Orinoco. Evaluación ecológica rápida de la fauna acuática. Informe final. INTECMAR, Universidad Simón Bolívar, Caracas, Venezuela. $644 \mathrm{p}$.

Brattegard, T. 1969. Marine biological investigations in the Bahamas 10. Mysidacea from shallow water in the Bahamas and southern Florida. Part I. Sarsia 39: 17-106.

Brattegard, T. 1970a. Marine biological investigations in the Bahamas 11. Mysidacea from shallow water in the Bahamas and southern Florida. Part II. Sarsia 41: 1-36.

Brattegard, T. 1970b. Mysidacea from shallow water in the Caribbean Sea. Sarsia 43: 111-154

Brattegard, T. 1973. Mysidacea from shallow water on the Caribbean coast of Colombia. Sarsia 54: 1-65.

Brattegard, T. 1974a. Additional Mysidacea from shallow water on the Caribbean coast of Colombia. Sarsia 57: 47-86.

Brattegard, T. 1974b. Mysidacea from shallow water on the Caribbean coast of Panama. Sarsia 57: 87-108.

Brattegard, T. 1975. Shallow-water Mysidacea from the Lesser Antilles and other Caribbean regions. Stud. Fauna Curacao 47: 102-115.

Camp, D.K., W.G. Lyons \& T.H. Perkins. 1998. Checklists of selected shallow-water marine invertebrates of Florida. Florida Mar Res. Inst. Tech. Rep. TR-3. St. Petersburg, Florida, EEUU. 239 p.

Capelo, J.C., J.V. Garcia \& G. Pereira. 2004. Diversidad de macroinvertebrados bentónicos del Golfo de Paria y delta del Orinoco, p. 55-60. In C.A. Lasso, L.E. Alonso, A.L. Flores \& G. Love (eds.). Rapid assessment of the biodiversity and social aspects of the aquatic ecosystems of the Orinoco Delta and the Gulf of Paria, Venezuela. RAP Bulletin of Biological Assessment 37. Conservation International. Washington DC, EEUU.

Castello, J. 1985. La ecología de los consumidores del estuario de la Lagoa dos Patos, Brazil, p. 383-406. In A. YánezArancibia (ed.). Ecología de comunidades de peces en estuarios y lagunas costeras. UNAM, México.

Cervigón, F. 1985. La ictiofauna de las aguas estuarinas del delta del río Orinoco en la costa atlántica occidental, Caribe, p. 56-78. In A. Yánez-Arancibia (ed.). Ecología de comunidades de peces en estuarios y lagunas costeras. UNAM, México.

Colonnello, G. 2004. Las planicies deltaicas del río Orinoco y golfo de Paria: aspectos físicos y vegetación, p. 37-54. In C.A. Lasso, L.E. Alonso, A.L. Flores \& G. Love (eds.). Rapid assessment of the biodiversity and social aspects of the aquatic ecosystems of the Orinoco Delta and the Gulf of Paria, Venezuela. RAP Bulletin of Biological Assessment 37. Conservation International. Washington DC, EEUU.

Díaz, J.M. 1995. Zoogeography of marine gastropods in the southern Caribbean: a new look at provinciality. Carib. J. Sci. 31: 104-121.

Díaz, Y.J. 2001. Contribución al conocimiento del Orden Amphipoda de la costa Centro Occidental de Venezuela. Tesis de Maestría, Universidad Simón Bolívar, Caracas. 542 p.

Díaz, Y.J. \& A. Martín. 2001. New records of amphipods (Crustacea: Amphipoda) from shallow water on the Caribbean coast of Venezuela. Rev. Biol. Trop. 49: 1271-1276.

Drooger, C.W. \& J.P.H. Kaasschieter. 1958. Foraminifera of the Orinoco-Trinidad-Paria shelf, p. 1-108. In N.V. Noord (ed.). Report on the Orinoco shelf expedition. Amsterdam, The Netherlands.

Escobar-Briones, E., I. Winfield, M. Ortiz, E. Gasca \& E. Suárez. 2002. Amphipoda, p. 341-371. In J. Llorente \& J.J. Morrone (eds.). Biodiversidad, taxonomía y biogeografía de artrópodos de México: Hacia una síntesis de su conocimiento. Volumen III. Comisión Nacional para el Conocimiento y Uso de la Biodiversidad. Facultad de Ciencias, UNAM, México.

Gûtu, M. 1996. The synoptic table and key to superespecific taxa of recent Apseudomorpha (Crustacea, Tanaidacea). Trav. Mus. Natl. Hist. Nat. Grigore Antipa. 36: 135-146. 
Keable, S. \& N. Bruce. 1997. Redescription of the north Atlantic and Mediterranean species of Natatolana (Crustacea: Isopoda: Cirolanidae). J. Mar. Biol. Assoc. UK 77: 655-705.

Kennish, M.J. 1986. Ecology of estuaries. Vol I. Physical and chemical aspects. CRC Press, Boca Ratón, Florida, EEUU.

Kensley, B. \& M. Schotte. 1989. Guide of the marine isopod crustaceans of the Caribbean. Smithsonian Institution Press, Washington DC, EEUU. 308 p.

Kosoy, N. 2001. Macrofauna asociada a los procesos de descomposición en Thalassia testudinum. Tesis de Licenciatura, Universidad Simón Bolívar, Caracas, Venezuela. 80 p.

Lasso, C.A., L.E. Alonso, A.L. Flores \& G. Love (eds.). 2004. Rapid assessment of the biodiversity and social aspects of the aquatic ecosystems of the Orinoco Delta and the Gulf of Paria, Venezuela. RAP Bulletin of Biological Assessment 37. Conservation International, Washington DC, EEUU. 358 p.

Ledoyer, M. 1986. Faune mobile des herbiers de phanérogames marines (Halodule et Thalassia) de la Laguna de Terminos (Mexique, Campeche). II. Les gammariens (Crustacea). An. Inst. Cienc. Mar Limnol. UNAM 13: 171-200.

Martín, J.W. \& G.E. Davis. 2001. An updated classification of the recent Crustacea. Natural History Museum of Los Angeles, Science Series 39. 124 p.

Martín, A., M. Ortiz \& Y. Díaz. 2002. Nuevos registros de anfípodos (Crustacea: Peracarida) para Venezuela. Bol. Invest. Mar. Cost. 31: 15-24.

Martín, A. \& Y.J. Díaz. 2005. Crustáceos peracáridos de la región atlántica de Venezuela: biodiversidad y taxonomía, p. 96-117. In M.G. Gómez, M. Capaldo, C. Yanes \& A. Martín (eds.). Frente Atlántico venezolano. Investigaciones geoambientales. Ciencias Ambientales. Tomo I. PDVSA-Fondo Editorial Fundambiente, Caracas, Venezuela.

McKinney, L.D., R.D. Kalke \& J.S. Holland. 1978. New species of amphipods from the western Gulf of Mexico. Contrib. Mar. Sci. 21: 133-159.

Méndez, J. 2005a. El delta del Orinoco, p. 12-24. In M.G. Gómez, M. Capaldo, C. Yanes \& A. Martín (eds.). Frente Atlántico venezolano. Investigaciones geoambientales. Ciencias de la Tierra. Tomo II. PDVSA-Fondo Editorial Fundambiente, Caracas, Venezuela.
Méndez, J. 2005b. Sedimentación en la plataforma continental y frente Atlántico del delta del Orinoco, p. 25-37. In M.G. Gómez, M. Capaldo, C. Yanes \& A. Martín (eds.). Frente Atlántico venezolano. Investigaciones geoambientales. Ciencias de la Tierra. Tomo II. PDVSA-Fondo Editorial Fundambiente, Caracas, Venezuela.

Myers, A.A. 1977. Studies on the genus Lembos Bate. VI. Atlantic species: L. dentischium sp. nov., L. kunkelae sp. nov., L. rectangulatus sp. nov., L. unicornis Bynum \& Fox. Boll. Mus. Civ. Stor. Nat. Verona 4: 125-154.

Myers, A.A. 1981. Amphipoda Crustacea. I. Familia Aoridae. Mem. Hourglass Cruises, 5: 1-75.

Ortiz, M. 1994. Clave gráfica para la identificación de familias y géneros de anfípodos del suborden Gammaridea del Atlántico Occidental Tropical. An. Inst. Invest. Mar. Punta Betín. 23: 59-101.

Ortiz, M. \& R. Lalana. 1993. Adición a la lista de especies y bibliografía de los anfípodos (Crustacea, Amphipoda) del Mediterráneo Americano. Rev. Invest. Mar. 14: 16-37.

Ortiz, M. \& R. Lalana. 1998. Lista actualizada de los crustáceos no decápodos de Cuba. Rev. Invest. Mar. 19: 92-99.

Pereira, G., J.V. García \& J.C. Capelo. 2004. Crustáceos decápodos del bajo delta del río Orinoco: biodiversidad y estructura comunitaria, p. 61-69. In C.A. Lasso, L.E. Alonso, A.L. Flores \& G. Love (eds.). Rapid assessment of the biodiversity and social aspects of the aquatic ecosystems of the Orinoco Delta and the Gulf of Paria, Venezuela. RAP Bulletin of Biological Assessment 37. Conservation International. Washington DC, EEUU.

Thomas, J.D. 1993. Identification Manual for the Marine Amphipoda (Gammaridea). I. Common coral reef and rocky bottom amphipods of South Florida. Florida Department of Environmental Protection. Division of Water Management. Final Report for DEP. Contract Number SP 290. Tallahassee, Florida, EEUU. $v+83$ p.

Thomas, J.D. \& J.L. Barnard. 1985. Perioculodes cerasinus, n. sp., the first record of the genus from the Caribbean Sea (Amphipoda: Oedicerotidae). Proc. Biol. Soc. Wash. 98: 98-106.

Thomas, J.D. \& R.W. Heard. 1979. A new species of Cerapus Say, 1817 (Crustacea, Amphipoda) from the northern Gulf of Mexico, with notes on its ecology. Proc. Biol. Wash. 92: 98-105. 
Sieg, J. \& R. Winn. 1978. Keys to suborders and families of Tanaidacea (Crustacea). Proc. Biol. Soc. Wash. 91: 840-846.

Winfield, I. \& M. Ortiz. 1996. Riqueza específica de crustáceos peracáridos en el Complejo Lagunar de Alvarado, Veracruz y sistemas circundantes. Rev. Zool. 2: 57-68.

Winfield, I., M. Ortíz, J. Franco \& C. Bedia. 1997. Distribución y diversidad del superorden Peracarida asociado a pastos marinos de Alvarado, Veracruz. Cuad. Mex. Zool. 3: 1-8.

\section{REFERENCIA DE INTERNET}

Kornfield, I., P. Haye \& C. Patel. 2001. Key to Families of Cumacea. University of Maine, Orono, Maine. (Downloaded: June 8, 2003, http://www.nature.umesci.maine.edu/Cumacea/key.html). 